\title{
Initiation of Excitation Waves: An Analytical Approach
}

\author{
VN Biktashev, I Idris \\ University of Liverpool, Liverpool, UK
}

\begin{abstract}
We consider the problem of initiation of a propagating wave in a one-dimensional excitable fibre. In the Zeldovich-Frank-Kamenetsky equation, a.k.a. Nagumo equation, the key role is played by the "critical nucleus" solution whose stable manifold is the threshold surface separating initial conditions leading to initiation of propagation and to decay. In ionic models of cardiac excitation fronts, the same role is played by the center-stable manifold of the "critical" front solution. Approximations of these manifolds by their tangent linear spaces yield analytical criteria of initiation. These criteria give a good quantitative appoximation for simplified models and a useful qualitatively correct answer for the ionic models.
\end{abstract}

\section{Introduction}

Existence of propagating wave solutions in cardiac excitation equations has been well studied, both numerically, and analytically for simplified models. However there are numerous applications where the important question is not whether the excitation wave can propagate, but whether it will be generated in response to a given initiation protocol. In heart, failure of initiation can cause or contribute to serious or fatal medical conditions, or render inefficient the work of pacemakers or defibrillators [1, chapters 31,52,53,95,96,98,105-110]. Mathematically, the question of whether a given stimulation will produce propagating wave involves non-stationary, spatially non-uniform solutions to a strongly nonlinear equation or system of equations. Any analytical progress in this direction is therefore rather difficult, and this problem is mostly approached numerically. We suppose that in view of the importance of the question, even crude analytical answers could be very useful.

\section{Methods}

Our approach is based on the understanding of the initiation problem in terms of dynamical systems theory, by treating the partial differential equation model (with one spatial dimension) as time-dependent ordinary differential equation in a functional space. From that viewpoint, the initiation problem reduces to description of the boundary of the basins of two attractors, of the resting state and of the propagating wave. It has been demonstrated $[2,3]$ that this boundary is the stable manifold of a saddle fixed-point called "critical nucleus", which is a spatially non-uniform unstable stationary solution $u_{*}(x)$ of the model. This approach has been used to design sophisticated numerical methods of determining the initiation conditions, e.g. [4]. We have recently demonstrated that for ionic models of cardiac excitation, the concept of the critical nucleus has to be replaced by the concept of "critical front" $\mathbf{u}_{*}\left(x-c_{*} t\right)$, and one has to consider center-stable rather than stable manifold, as the boundary of the basins of attraction $[5,6]$.

Here we use this theoretical background to suggest a practical method of studying the initiation problem. The leading idea is to replace the (center)-stable manifold with its linear approximation, the (center)-stable space. This implies linear approximation of the partial differential equations of the model around the critical solution, i.e. critical nucleus or critical front as appropriate. In this respect, our method is different from other theoretical approaches, which used linear or nonlinear expansion around the resting state [7] or Galerkin-style approximation to arbitrarily chosen finite-dimensional manifold of the phase space [8].

As test examples for our approach we consider Zeldovich-Frank-Kamenetsky (ZFK) equation [9] also known as Nagumo equation [10],

$$
u_{t}=u_{x x}+f(u), u(u-\theta)(1-u), 0<\theta<1 / 2,
$$

as the archetypical bistable reaction-diffusion equation, and the simplified piecewise-linear model of $I_{\mathrm{Na}}$-driven cardiac excitation fronts

$$
\begin{aligned}
& \mathbf{u}_{t}=\mathbf{D} \mathbf{u}_{x x}+\mathbf{f}(\mathbf{u}), \mathbf{u}=\left[\begin{array}{l}
E \\
h
\end{array}\right], \mathbf{D}=\left[\begin{array}{ll}
1 & 0 \\
0 & 0
\end{array}\right], \\
& \mathbf{f}=\left[\begin{array}{l}
h \Theta(E-1) \\
(\Theta(-E)-h) / \tau
\end{array}\right], \tau>\tau_{\min } \approx 7.6740
\end{aligned}
$$

suggested in [11]. The advantage of these two models is that they represent typical qualitative features of the corresponding classes of more accurate models, and at the same time admit analytical solutions, both for the propagating 


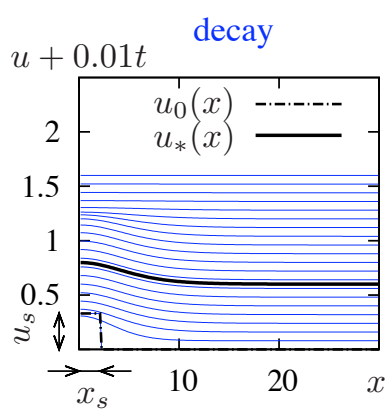

(a)

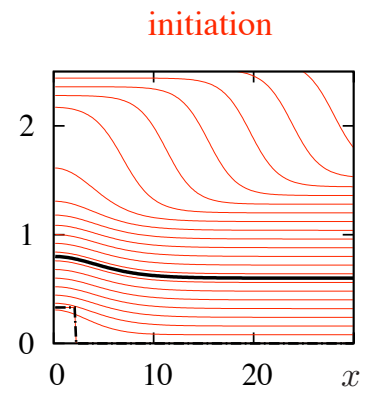

(b)

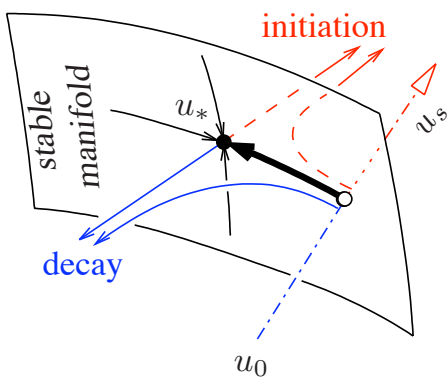

(c)

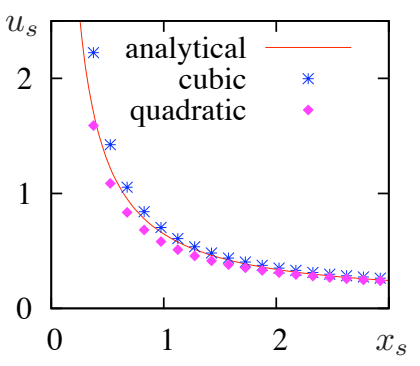

(d)

Figure 1. (color online) Initiation of bistable fronts. $(a, b)$ Response to an below- and above-threshold initial perturbation in ZFK equation, (1) for $\theta=0.13$. Blue/red thin solid lines: voltage profiles at regular time intervals, shifted upwards with time. Dash-dotted black lines: initial conditions, bold solid black lines: the critical nuclei. (c) The sketch of a stable manifold of the critical nucleus $u_{*}$. The critical nucleus is represented by the black dot; the critical trajectories, constituting the stable manifold, are shown in black. The family of initial conditions is represented by the dash-dotted line. The bold black line is the critical trajectory with initial condition in that family. The sub-threshold trajectories coming towards the reader are represented by the blue line, while the red lines going away from the reader represent super-threshold trajectories. Note that the point where the initial conditions intersect the stable manifold is shown as the empty circle. (d) The critical "strength-extent" curve separating initiation and decay initial conditions. The two numerical curves correspond to the original cubic nonlinearity in (1) and its quadratic approximation (3).

fronts, and for the critical solutions, i.e. the cricial nucleus of (1) and the critical front for (2). The knowledge of the analytical expression for the critical solution is of course very handy for bulding a linear approximation around it.

We consider the problems on half-fibre, $x \geq 0$, with noflux conditions on $x=0$. As an initiation protocol, we consider instant spatially-extended perturbation at $t=0$ raising the voltage from the resting state,

$$
u(x, 0)=u_{s} \Theta\left(x_{s}-x\right)
$$

for (1) and

$$
\mathbf{u}(x, 0)=\left[\begin{array}{l}
-\alpha \\
1
\end{array}\right]+E_{s} \Theta\left(x_{s}-x\right)\left[\begin{array}{l}
1 \\
0
\end{array}\right], \alpha>0,
$$

for (2). Extension to a stimulus with finite temporal duration is possible [12] but will not be considered here.

\section{Results}

\subsection{Bistable fronts}

Fig. 1(a-c) illustrates the concept of the critical nucleus, as a universal transient observed with any near-threshold condition, and the role of its stable manifold as the boundary of the basins of attractors. In the limit of $\theta \ll 1$, the nonlinearity can be approximated by a quadratic polynomial,

$$
f(u) \approx u(u-\theta),
$$

and then the critical nucleus has the form

$$
u_{*} \approx \frac{3}{2} \theta \operatorname{sech}^{2}(x \sqrt{\theta} / 2) .
$$

We consider the linearized equation for $v(x, t)=u(x, t)-$ $u_{*}(x)$. The stable manifold is defined by the equation that the projection of the initial condition $v(x, 0)$ on the unstable direction vanishes,

$$
\int_{0}^{\infty} \phi_{1}(x)\left(u_{s} \Theta\left(x_{s}-x\right)-u_{*}(x)\right) \mathrm{d} x=0,
$$

where

$$
\phi_{1}(x)=C_{1} \operatorname{sech}^{3}(x \sqrt{\theta} / 2), C_{1} \neq 0,
$$

is the eigenfunction of the self-adjoint linearized operator, corresponding to the only positive eigenvalue $\lambda_{1}=5 \theta / 4$. This leads to the equation of the critical curve $u_{s}\left(x_{s}\right)$ of the form

$$
\begin{array}{r}
u_{s}=\frac{9 \theta}{8}\left[\frac{2}{\pi} \tanh \left(\frac{x_{s} \sqrt{\theta}}{2}\right) \operatorname{sech}\left(\frac{x_{s} \sqrt{\theta}}{2}\right)\right. \\
\left.+\frac{4}{\pi} \arctan \left(e^{x_{s} \sqrt{\theta} / 2}\right)-1\right]^{-1} .
\end{array}
$$

Fig. 1(d) demonstrates the accuracy of this analytical approximation. We see that the error introduced by replacing the stable manifold with the stable space is of the same order of magnitude as the error caused by the quadratic approximation of the function $f(u)$.

\subsection{Cardiac fronts}

Fig. 2(a-c) illustrates the concept of the critical front, as a universal transient observed with any near-threshold 


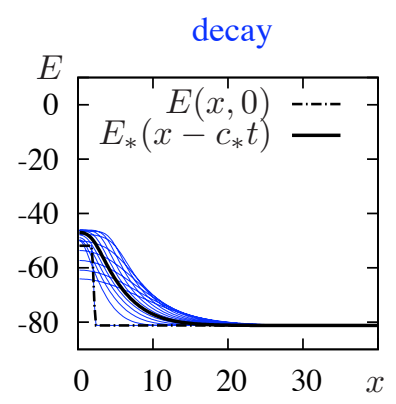

(a)

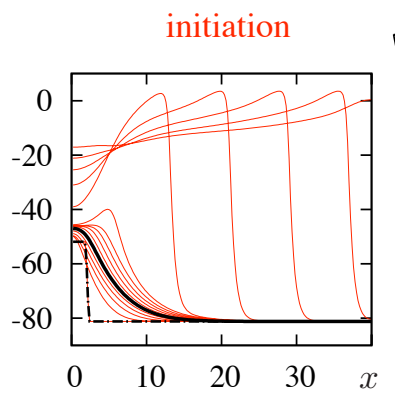

(b)

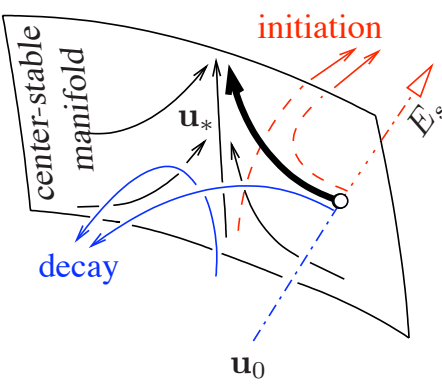

(c)

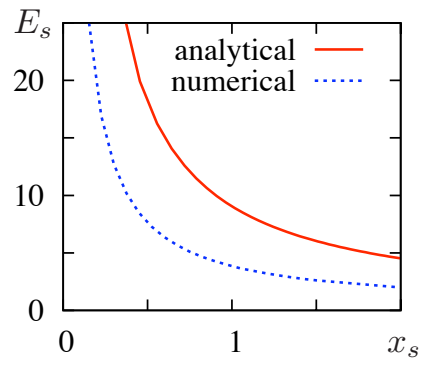

(d)

Figure 2. (color online) Initiation of cardiac fronts. (a,b) Response to an below- and above-threshold initial perturbation in Courtemanche et al. model [13]. Blue/red thin solid lines: voltage profiles at regular time intervals. Dash-dotted black lines: initial conditions, bold solid black lines: the critical fronts at a selected time moment. (c) The sketch of a stable manifold of the critical front $\mathbf{u}_{*}$. The critical front is represented by the black nearly straight upward trajectory; the critical trajectories, constituting the center-stable manifold, are also shown in black. The family of initial conditions is represented by the dash-dotted line. The bold black line is the critical trajectory with initial condition in that family. The sub-threshold trajectories coming towards the reader are represented by the blue line, while the red lines going away from the reader represent super-threshold trajectories. Note that the point where the initial conditions intersect the center-stable manifold is shown as the empty circle. (d) The critical "strength-extent" curve separating initiation and decay initial conditions in the simplified model (2).

condition in ionic cardiac models, and the role of its centerstable manifold as the boundary of the basins of attractors. The specifics of the critical front is that it does not satisfy the no-flux boundary condition at $x=0$ so the problem has to be considered on the whole line $-\infty<x<\infty$. In such setting, the linearized operator has a zero eigenvalue, corresponding to the equivariance with respect to the translations along the $x$ axis. A closely related fact is that critical fronts make a one-parametric family $\mathbf{u}_{*}^{\Delta}(x, t)=$ $\mathbf{u}_{*}\left(x-c_{*} t-\Delta\right)$ with an arbitrary parameter $\Delta$, and when performing linearization $\mathbf{v}(x, t)=\mathbf{u}(x, t)-\mathbf{u}_{*}^{\Delta}(x, t)$ about it, one has to make a decision which $\Delta$ to choose. A natural choice is to demand that the projection of $\mathbf{v}(x, 0)$ on the neutral direction along the zero-value eigenfunction vanishes, too, as well as the projection on the unstable direction. This leads to system of two simultaneous equations relating $x_{s}, E_{s}$ and $\Delta$,

$\int_{-\infty}^{\infty} \boldsymbol{\psi}_{j}(x-\Delta)\left(\mathbf{u}(x, 0)-\mathbf{u}_{*}(x-\Delta)\right) \mathrm{d} x=0, j=1,2$

where the initial condition $\mathbf{u}(x, 0)$ is assumed evenly extended to $x<0, \psi_{1,2}(x)$ are projectors on the unstable eigenfunction $\phi_{1}(x)$ corresponding to a $\lambda_{1}>0$ and on the translational eigenfunction $\phi_{2}(x)=\partial_{x} \mathbf{u}_{*}(x)$ corresponding to $\lambda_{2}=0$. Note that the linearization operator in this case is not self-adjoint therefore $\phi_{j} \neq \boldsymbol{\psi}_{j}$. For the simplified model (2), the critical front solution

$$
\begin{aligned}
& \mathbf{u}_{*}(x)=\left[\begin{array}{l}
E_{*}(x) \\
h_{*}(x)
\end{array}\right] \text { has the form [11] } \\
& E_{*}(x)= \begin{cases}-\alpha+\alpha \exp \left(-c_{*} x\right) & (x \geq-\xi), \\
\omega-\frac{\tau^{2} c_{*}^{2}}{1+\tau c_{*}^{2}} \exp \left(\frac{x}{\tau c_{*}}\right) & (x \leq-\xi),\end{cases} \\
& h_{*}(x)= \begin{cases}1 & (x \geq 0), \\
\exp \left(\frac{x}{\tau c_{*}}\right) & (x \leq 0),\end{cases}
\end{aligned}
$$

where $\omega=1+\tau c_{*}^{2}(\alpha+1), \xi=\frac{1}{c_{*}} \ln \left(\frac{1+\alpha}{\alpha}\right)$ and $c_{*}$ is an implicit function of $\tau$ and $\alpha$ defined as the smallest of the two solutions of the transcendental equation

$$
\tau c_{*}^{2} \ln \left(\frac{(1+\alpha)\left(1+\tau c_{*}^{2}\right)}{\tau}\right)+\ln \left(\frac{\alpha+1}{\alpha}\right)=0 .
$$

This analytical solution for $\mathbf{u}_{*}$ allows an analytical solution for the projectors $\psi_{j}$ required in (7). The calculations involved are, however, rather extensive to be presented here in full, so we refer the interested reader for further details to [14] or to our future more detailed publications, and present here only the the outline of the method. The linearized operator is defined in piecewise manner in the three intervals $I_{1}=\{x \leq-\xi\}, I_{2}=\{-\xi \leq x \leq 0\}$ and $I_{3}=\{x>0\}$, with singularities at $x=-\xi, 0$ due to linearization of the Heaviside functions. Inside each of the three intervals, the linearized operator has constant coefficients, so the eigenfunctions have the form $\phi_{j}(x)=$ $\left\{\boldsymbol{\phi}_{j l}(x), x \in I_{l}\right\}, \phi_{j l}=\sum_{k=1}^{3} a_{j k l} e^{\nu_{j k l} x}, l=1,2,3$, where $\nu_{j k l}$ is the $k$-th eigenvalue of a $3 \times 3$ matrix of the 
constant coefficients in the interval $I_{l}$, and index $j$ enumerates the eigenvalues $\lambda_{j}$ of the linearized operator. The matching conditions for the pieces of $\phi_{j, l}(x), l=1,2,3$ at $x=-\xi$ and $x=0$ lead to a transcendental characteristic ("Evans") equation for $\lambda_{j}$, which has been investigated in [15]. In particular, for $\alpha=1$ and $\tau=8.2$ we have $c_{*} \approx 0.33187$ and the only eigenvalue with a positive real part is $\lambda_{1} \approx 0.039903$. Besides, we have of course the eigenvalue $\lambda_{2}=0$ corresponding to the translation mode. Similarly, we have $\boldsymbol{\psi}_{j}(x)=\left\{\boldsymbol{\psi}_{j l}(x), x \in I_{l}\right\}$, $\boldsymbol{\psi}_{j l}=\sum_{k=1}^{3} b_{j k l} e^{\mu_{j k l} x}, l=1,2,3$, the matching conditions for which lead to the same characteristic equation as for $\phi_{j}$. After we substitute the thus found $\boldsymbol{\psi}_{j}(x), j=1,2$ into (7), we obtain a closed system of two finite equations relating $x_{s}, E_{s}$ and $\Delta$. It is linear with respect to $E_{s}$ which is therefore easily eliminated, leaving a single finite transcendental equation relating $\Delta$ with $x_{s}$ and thus implicitly defining $E_{s}$ as the function of $x_{s}$. This dependence $E_{s}\left(x_{s}\right)$ is the sought for critical strength-extent curve. The comparison of this analytical prediction with the direct numerical simulations is presented on Fig. 2(d).

\section{Discussion and conclusions}

The proposed method uses linearization around a critical solution so it should work when the initial condition is not too far from the critical solution, with a suitable shift in space for the case of a critical front. Exactly how far it should be for the method to work is not obvious a priori. The considered examples show that the method works surprisingly well even for such crude initial conditions as stepwise profiles.

Practical interest presents the possibility of extending the approach developed using the above archetypical, "toy" models to more realistic models describing concrete systems. We expect that the method work in the same way, only the critical solution, as well as the projectors to its principal eigenfunctions are to be calculated numerically. Notice that the case of critical front would be more complicated as we would need projectors of two eigenfunctions. After that, equation (5) is linear so readily solvable for $u_{s}$, whereas system (7) is nonlinear with respect to the unknown $\Delta$ so will need numerical solution again.

As already noted, the method readily generalizes to the stimulation extended in time, which is more usual in electrophysiological applications. The critical nucleus case then easily leads to the classical Lapicque-Blair-Hill equation of the strength-duration curve [12], but the critical front case is again more complicated.

\section{Acknowledgements}

This study has been supported by EPSRC (grant GR/S75314/01) and MacArthur Foundation.

\section{References}

[1] Zipes DP, Jalife J (eds.). Cardiac electrophysiology: From cell to bedside. Philadelphia: W B Saunders Co, 2004.

[2] McKean HP, Moll V. A threshold for a caricature of the nerve equation. Bull AMS 1985;12(2):255-259.

[3] Flores G. The stable manifold of the standing wave of the Nagumo equation. J Diff Eq 1989;80:306-314.

[4] Moll V, Rosencrans SI. Calculation of the threshold surface for nerve equations. SIAM J Appl Math 1990;50(5):1419_ 1441 .

[5] Idris I, Simitev RD, Biktashev VN. Using novel simplified models of excitation for analytic description of initiation propagation and blockage of excitation waves. Computers in Cardiology 2006;33:213-216.

[6] Idris I, Biktashev VN. Critical fronts in initiation of excitation waves. Phys Rev E 2007;76(2):021906.

[7] Suarez Antola RE, Sicardi Schifino AC. A modal approach to threshold dynamics for excitable tissues stimulated by external electrodes: I: one state variable model for a fiber. Physica D 1996;89(3-4):427-438.

[8] Neu JC, Preissig RS, Krassowska W. Initiation of propagation in a one-dimensional excitable medium. Physica D 1997;102:285-299.

[9] Zel'dovich YB, Frank-Kamenetsky DA. Towards the theory of uniformly propagating flames. Doklady AN SSSR 1938; 19:693-697.

[10] McKean Jr. HP. Nagumo's equation. Adv Appl Math 1970; 4:209-223.

[11] Biktashev VN. Dissipation of the excitation wavefronts. Phys Rev Lett 2002;89(16):168102.

[12] Idris I, Biktashev VN. An analytical approach to initiation of propagating fronts. submitted, 2008. See http://arxiv.org/abs/0809.0252.

[13] Courtemanche M, Ramirez RJ, Nattel S. Ionic mechanisms underlying human atrial action potential properties: insights from a mathematical model. Am J Physiol 1998; 275:H301-H321.

[14] Idris I. Initiation Of Excitation Waves. Ph.D. thesis, University of Liverpool, 2008. See http://www.maths.liv.ac.uk/ vadim/theses/Idris.pdf.

[15] Hinch R. Stability of cardiac waves. Bull Math Biol 2004; 66(6):1887-1908.

Address for correspondence:

V. N. Biktashev

Department of Mathematical Sciences

University of Liverpool

Liverpool L69 7ZL

UK

E-mail address vnb@liv.ac.uk 